\title{
The Action Mechanism of Chemical Agents in the Differences Between Oral Pathologyand General Pathology
}

\author{
ION SARBU ${ }^{1}$, CRISTINA POPA ${ }^{3}$, VICTOR COSTAN ${ }^{2}$, ADRIAN BEZNEA $^{3 *}$, GABI TOPOR $^{3 *}$, \\ CATALIN PLESEA CONDRATOVICI ${ }^{3}$, SILVIA FOTEA ${ }^{3}$ \\ ${ }^{1}$ Gr. T. Popa University of Medicine and Pharmacy, Faculty of Dental Medicine, 16 Universitatii Str., 700115, Iasi, Romania \\ ${ }^{2}$ Carol Davila University of Medicine and Pharmacy, Faculty of Dental Medecine, 37 Dionisie Lupu Str.,020021, Bucharest, \\ Romania \\ ${ }^{3}$ Dunarea de Jos University of Galati, Faculty of Medicine and Pharmacy, 47 Domneasca Str., 800008, Galati, Romania
}

The pathological anatomy proves to be indispensable for the correct diagnosis and treatment, scientific and for the formation of the medical thinking. In the conditions of the great diversity of the oral pathology, the utility of the anatomo-pathological investigation is confirmed by its possibilities to elucidate the structural, material basis of the quasi-totality of the diseases in this field and to differentiate them on this basis, providing a conclusive diagnosis. Oral pathology problems frequently occur in medical practice, and doctors often face this type of condition. Risk factors for oral pathology include: poor nutrition and hygiene, trauma, stress, smoking, alcohol and other illicit drugs. The lesions in the oral cavity must be seen in the light of the pathological processes of the system and correlated with the diseases of the system, because the diseases of the system produce oral manifestations and conversely - the oral diseases produce system manifestations.The oral health status of the target population groups is monitorised worldwide and associated with selected chronic diseases as well as common risk factors. Our study includes 112 patients, who were referred by dentists, family doctors, dermatologists, ENT specialists, BMF surgeons. Histopathology techniques for oral pathology pieces are processed in a similar way to the pieces of general pathology. In order to develop effective prevention and treatment measures when needed, mucosal disorders should be understood in terms of their distribution, etiology and epidemiological characteristics.

Keywords: anatomo pathological histology, oral pathology, pathology general health.

In the so-called general pathology, the center of gravity must fall precisely on the experimental pathology, on the analysis of the pathological processes on the experimental path. In order to understand the life of a sick organism, it is necessary to know the morphological changes in different stages of pathological processes development. Morphopathology deals with the study of morphological changes in a sick organism.Both disciplines, that is, both the pathophysiology and the morphopathology, complement each other and constitute a vast field of medical knowledge, that is to say the so-called pathology The pathophysiology uses the data of the morphological changes found as a result of the pathological processes and is closely related to the pathological anatomy, which deals with the same problems, but from another point of view; both have the same object of study, namely, the sick human body.Any pathological action causes from the very beginning a defense reaction of the organism, directed against the injurious agent or against the injury[1]. The pathological phenomena are recognized not only by studying the functional changes, the organization and its various parts. Given the unity of function and form, it is necessary to study morphological changes. Pathology is an important tool for medicine to understand the mechanism, the changes of the organ produced by the diseases, to improve the diagnosis, not only during the life but after the patient's death. It is an important aspect that can be established only by means of biopsy pathology (the malignant or benign nature of a tumor); it comprises two branches: the pathological anatomy, which examines the changes of the macroscopic organs, and the histological pathology that examines the changes of the diseased tissues or cells under the microscope. Oral pathology is a border specialty between dentistry and general medicine, mainly dealing with the diagnosis and treatment of oral mucosal disorders. Most of the conditions are located in the mouth mucosa: cheeks, tongue, palate; it is a complex pathology, which frequently requires anatomopathological examinations, complex laboratory investigations, radiology and surgery. Any disease appears as a result of the disturbance of the correlations between the body and the environment. The fair interpretation of the etiology of diseases is possible only when the laws of these correlations are discovered. The cause determines the specific features of the pathological process it causes.

*email: adrianbeznea@hotmail.com; gabi_topor_atu@yahoo.com 
Establishing the connection between the cause and the ongoing pathological process is necessary for the treatment and prophylaxis of different diseases. The causes of the diseases are most often pathogens from the external environment that exert their action on the body; in such cases, we talk about the external (exogenous) causes of the diseases. The causes of the diseases that appear in the respective organism are called internal causes (endogenous)[2].

The etiology of pathological processes must not be to oppose these two elements to one another. Due to the unity existing between the external and the internal elementary, the pathogenic factor cannot be considered isolated from the physiological properties of the respective organism. Having received the living organism, capable of reacting, external actions pathogenic causes disturbances in its internal environment, which in turn can become cause of a new pathologic process. For example, adiposity arising from the disorder of blood circulation food can become an internal issue for blood circulation disorder; the bleeding caused by a traumatic action can in turn become an internal cause of an inflammatory reaction. Thus, the internal elemental should be considered as the result of receiving the external element.

The agents are different excitants or obviously harmful excitators, which from the external environment exert their action on the organism. The pathogenic character of the excitants is also determined by the functional state of the organism on which these excitators act.

Due to the theory of conditioned reflexes, the circle of etiological factors of the diseases has widened due to a large number of weak or indifferent exciters. These excitants, under certain conditions, after a repeated association with the action of the non- uncondensed patogenic agents, may become conditioned, being able to cause the appearance of these conditions according to the principle of reflex-conditioned connections.

In each case of disease, it is necessary to isolate the predominant importance in the origin of this disease of the exciting pathogen that acts outside or of the disturbance of the properties of the organism itself, of its capacity to react to a non-action, even of an indifferent exciter.

The causes of the pathological processes are often the factors of the external environment. The properties of the organism, formed in turn in the correlations with the environment, have in this case a role of conditions that favor the appearance of diseases.The results produced in the organism by pathogens may vary depending on the appropriate conditions, because the cause of the pathological process never acts in isolation, but always according to the external and internal environment conditions of the organism.

It is understood, under the etiology of the pathological process, both the cause and the whole complex of conditions in the presence of which the pathogen manifests its action on the organism, causing the pathological process.

The study of the pathological processes should not be limited to the elucidation of the cause that caused a certain process. All this must be of interest to us and the question of how the pathogen acts on the organism or what is the mechanism of the pathological process. Clarification of the mechanisms of appearance and development of pathological processes and diseases is the task of pathogenesis. It is closely related to the etiology[3].

The pathogenic agent, acting on the organs of the smoker, first of all causes the excitation of the receptors, whose sensitivity exceeds the threshold of sensitivity of other tissue elements several times. The development of the inflammation process in a portion of the tissue is greatly attenuated after sectioning of the related nerves or after stopping their action by means of anesthetic substances. In case of inflammation, besides the tissue damage and its metabolism disorder, under the influence of reflex activity, defense phenomena appear, namely: leukocyte reaction, phagocytosis and cell proliferation.

Due to these processes, the affected functions are rehabilitated and the injury caused is eliminated.

Pathological processes can occur, both after the mechanism of the unconditioned and the conditioned reflexes. Depending on the etiological factor, its place of application and the properties of the organism, the pathogenesis of different diseases may be related to the function of the various segments of the nervous system, from the peripheral endings of the centripetal nerves to the cerebral cortex.

Disorders of correlations between the central nervous system and the internal environment of the body occupy an important place in the pathogenesis of diseases.

Because the disorder of the tissue metabolism is the main component of any pathological process, it is natural for the conception of a certain importance of the trophic function of the nervous system in the pathogenesis of diseases to appear.

The pathological processes are not strictly localized. Due to the integrity of the organism and the interaction of its component parts, regulated by the activity of the nervous system, so-called local processes cannot be considered broken by the whole organism; in general, these processes are a local expression of a general disease. At the same time, the pathological process, appearing in one part or another of the organism, becomes at a certain stage of its development starting point of new relations of the local element, compared to the general one, reflexively exercising its action on the whole organism[4].

The outbreak of inflammation can significantly alter the sensitivity of the whole organism to heterogeneous proteins and to infectious agents.Disease pathogenesis research study requires all stages of development of pathological phenomena, to replace them and the link between them.

Chemicals can carry out different actions and are often causes of illness, called poisoning. Poisons can be caused, both by substances that enter the body (exogenous toxins) and by substances that form in the body itself (endogenous toxins)[5]. 
The appearance of pathological processes due to the action of the chemical substances depends on their dose, their solubility in biological moods, their mode of administration as well as the resistance of the body. One and the same chemical exerts, depending on the dose, a curative action, a toxic effect and even deadly poisoning. Among the toxic substances are toxins of inorganic and organic origin.

Inflammation is part of the most widespread pathological processes. Studying the mechanism of the onset and development of inflammation contributes to clarifying the essence of this process.

Inflammation is a complex reflex reaction of the organism, against the action of some harmful agents, reaction that manifests itself in the form of a complex of functional and structural changes of vascular-tissue nature, this reaction appeared in the process of evolution and is characterized by three main forms, which are closely related to each other and evolve simultaneously. The inflammatory process appears in different ways; inflammation occurs in response to a tissue injury.

The oral cavity together with the tongue and teeth are indicators of the status of systemic function, of the whole body, because at this level the symptoms of countless diseases may manifest or appear.The most common diseases of the oral cavity are cavities, periodontitis, oral cancer, oral infectious diseases, trauma or hereditary lesions.

The pathology of the oral cavity can be highlighted by different diagnostic modalities depending on the signs and symptoms that can predict the definitive diagnosis: physical examination of the structures of the oral cavity, teeth and gums; dental sensitivity inspection; lymph node inspection; complete hemolysogram and VSH HIV serological test; immunoglobulin test; X-ray and CT scan; oral secretion sampling for microbiological examination, biopsy, puncture.

Of all the medical specialties, in the sphere of dentistry these disorders are most often encountered. Often, however, the dentist escapes the presence of these changes, either because of the difficulty generated by the anxiety present sometimes in the young patient[6-8].

The reason lies in the increased use of the more extensive of harmful factors: alcohol, tobacco, drugs, nutrition based on synthetic chemical compounds whose use is currently age meets the increasingly early. At the global level, the general human pathology changes and is reflected in this field, in very different, very misleading forms. It is important for dentists, dermatologists, BMF surgeons, ENT surgeons to know this changing, simulating border pathology, there are very high risks of treating a patient without being aware of the existence of a severe systemic underlying disease.The lesions in the oral cavity must be seen in the light of the pathological processes of the system and correlated with the diseases of the system, because the diseases of the system produce oral manifestations and conversely - the oral diseases produce system manifestations. Oral cavity - the incipient segment of the digestive tract can present marked lesions in many general diseases due to the disorders present in these diseases[9-11].

Irritation of the oral mucosa by eliminating endogenous toxic products from saliva (urea, uric acid, creatinine, ammonia, ammonium carbonate) also contributes at the formation of these lesions.Salivary flow disorders (hyposalgia, asialia, xerostomia, etc.) and changes in salivary $\mathrm{pH}$ are favorable conditions for increasing microbial virulence as well as the biological balance of the oral environment.

These lead to the accentuation of the oral-dental lesions as well as the appearance of complications of proximity or distance[12-14].

As precancerous conditions, we can mention both some inflammatory processes with dysplasia, as well as some disembryoplastic tumors, with different degrees of malignant potential, giving special importance to pre-invasive cancer.The first category includes: actinic cheilitis, Paget and Bowen diseases, flat lichen and leukoplakias with their various etiopathogenic forms and to a certain extent epulis.

Actinic cheilitis is an inflammation of the lips due to the action of the sun rays, the redness of the lips being a photosensitizer; it appears as a diffuse thickening of the injured region, with bleeding cracks and painful ulcers. Macroscopic: ortho- and hyperparakeratosis, basophilic degeneration of the papillary body and lymphoplasmocyte infiltrate.

Long-lasting evolution; it may be malignant (epidermoid carcinoma, usually spinocellular).

Bowen's disease is a precancerous dermatosis; it develops on the skin and on the Malpighian mucosa; is an anplasia dysplasia, considered by some authors to be an 'in situ' carcinoma; it evolves into an epidermoid carcinoma.

Paget's disease of the lips: dyskeratosis with parakeratosis, segregation by desmolysis, ballooning degeneration of epithelial cells and lymphoplasmocyte infiltrate; it can be malignant (spinocellular carcinoma). There are also some oral signs, namely: the ' gall' sign. If the facial massif is affected, the mandible is especially interested, taking the appearance of 'gall', with bone hypertrophy and enlargement of the dental alveoli, which leads to the spontaneous fall of teeth.

Another sign is the 'too small hat sign', which consists in increasing the volume of the skull box (too small hat, signe du chaupeau ') in aesthetic disagreement with the facial massif.

The flat lichen, which is in particular a dermatological condition, may also have oral localization, under the three main forms (non-erosive, bulbous and ulcerated); it is possible to become malignant, turning into epidermoid carcinoma, which does not happen in the dermatological locations. 
Oral lichen appears most commonly inside the cheeks, but can also affect the tongue, lips, and gums. The typical oral plane lichen appears in the form of some fine white lines and dots that usually do not cause symptoms. Dentists often discover them during routine dental examen.

Severe forms of oral lichen can cause painful wounds and ulcers in the oral cavity. To confirm the diagnosis may be necessary a pre elevation biopsy.

Sometimes, the tissue taken must be studied by a complicated technic and blood tests could be necessary to exclude other diseases.

There are cases of flat lichen as an allergic reaction to dental materials, but they are very rare.

Allergenic tests may be needed to identify the allergy; in this case, removing the dental material is recommende .

Leukoplakias are an important condition of the oral mucosa due to its frequency and the possibility of malignancy; microscopic: hypertrophy of the epithelium as a whole, with an exaggeration of the sinuosity produced by the entanglement of the papillae and the interpapillary buds; the surface is covered by a thick layer of horny blades, with numerous granules of keratohyalin and eleidine; they are divided into four groups: the last group presents a disorganization of the tissue architecture, as well as the cariatotypes, being an intraepithelial carcinoma.Oral leukoplakia complicated means white or gray spots located in the oral cavity (on the tongue, under it or inside the cheeks). The word "leukoplakia" comes from the Greek noun "leukoplakia", which means "white spot" or "white formation" (euko = white + plakos = stain).

This condition can also be found under other names: leucokeratosis, idiopathic leukoplasty and idiopathic keratosis or, white idiopathic plaque.

The condition is manifested by covering with keratosis or adherent plaque of large areas of the oral cavity, respectively on the mucous membrane of the mouth. For this reason, when leukoplakia occurs not only in the teeth, but also on other surfaces of the mouth, the correct name is oral leukoplakia.

Epulis: may be pseudotumeral (peripheral granuloma), but the myeloplasmic and papillomatous giant cell form represent true tumors of mesenchymal or epithelial origin, with potential for sarcomatous malignancy, or epidermoid carcinomatosis (especially spinocellular), taking on a character alveolar bone (osteoclasia).

Under the term epulides, the proliferative, tumorous, slow-evolving forms localized to the gum are described; occur as a reaction to infectious, toxic, chronic local irritants, tartar maintenance, root residues, unsuitable prosthetic work, repeated minor trauma.

\section{As dysembryoplastic tumors we have:}

Hamartoma: a benign tumor or a malignant hamartoblastoma develops as a consequence of defects in the embryonic development, with the formation of epithelial and mesenchymal tissue combinations, with an increase of one's self confidence; oral localization: thyroid follicles in tongue, lymphoid follicular tissue in the extraganglionic region.

Coristoma: an embryonic tumor with a development of corists (embryonic fragments), observing the existence of tissues that normally do not exist in the oral cavity (sebaceous glands in the lamina propria of the oral mucosa - Fordyce-cartilage granules in the body of the mandible in adulthood, or neural cells); questionable malignancy.

The branchial cyst usually with locvalization in the anterior portion of the sternocleidomastoid muscle; delimitation of the cyst is done by squamous epithelial cells, having around connective tissue and imfoid follicular structures; it may have as a starting point remnants of pharyngeal sacs, or it results from a cystic degeneration of epithelial embryonic inclusions in the lymph nodes; possible malignancy on the epithelial line.

Papillary cystadenolymphoma: particular tumor of the salivary glands; usually benign; histogenesis is confusing (according to Harris it would be heterotypic salivary glands included in lymphatic islands). Microscopic it is an association of epithelial tissue with acins and cystic dilations, with papillary proliferation, with lymphoid tissue with follicular structures; they may be areas of malpighian metaplasia; it is possible on both lines.

Pleomorphic adenoma: tumor of the main salivary glands and accessory (80\% in the parotid); slow growth in the form of well-delimited, single or multiple nodules. The histological picture is complex, polymorphic and specific to this tumor; it may have a tubular structure, characterized by a uniform distribution of the cubic cells, presenting vacuoles and prominent nuclei (as myoepithelial cells of the normal gland), or similar to ductal cells (eosinophilic cells with granular cytoplasm), or fibrroadenoma-like structure mucoid (cylindromatosus), myxoid, chondroid or osteoid; the level of the glandular epithelium and areas of epidermoid metaplasia can be observed.

Potentially relapsing and malignant degeneration on the epithelial line, with anaplasia and atypical mitoses (adenocarcinoma, more rarely, epidermal carcinoma and epidermoid carcinoma). Ganglion metastases are common; distant metastases may occur in the lungs, bones, etc

Oncocytoma: tumor of the salivary glands (parotid) characteristic of old age; it is benign and rare; it may be pluricentric, but this does not indicate any malignancy. Microscopic: large and clear cells (Hamperl), thus distinguished by the enzinophilic adenoma. The origin of oncocytes is not clear (they are found both in acini and in the canal). Cell proliferation is slow. 
Follicular cyst: benign tumor of the jaws, with a series of theories and on pathogenesis (Broca's follicular theory, representing a teratological developmental disorder, or Malassez's gubernacular theory): a unity of views has not been reached. Microscopic: the cystic membrane consisting of a malpighian epithelial layer, bordered by a variable density connective tissue. Carcinomatous malignancy is exceptional and occurs after recurrence.

Ameloblastoma is a heterotopy of the jaws (Broca, 1868); the term adamantinoma was introduced by Malassez (1885); approximately $1 \%$ oral tumors $(80 \%)$ in the mandible.

It grows slowly, with minor symptoms, or even asymptomatic, being able to be discovered at a routine dental examination; other times, however, it leads to bone deformities, the radiological examination showing a lysis of bone tissue, mono- or polycystic. Microscopic may have follicular aspects with cystic dilatations, or compact forms in the form of epithelial cell bundles, in a more or less dense stroma; there are also mixed forms. The histological table is pathognomonic due to the existence of small and tachychromatic cells and large clear cells, palisadically arranged. Malignancies rarely occur in epidermoid carcinoma[14].

As malignant tumors we mention in the order of the frequency lip cancer (especially in the lower lip); it is usually spinocellular epidermoid carcinoma; metastasis is submandibular lymphoganglionic; may be bilateral, due to crossing paths lymphatic submentonian, tonguecancer, also in epithelial line, evolving very serious lips malignant melanoma, adenocarcinoma parotid form with invasion upon the facial nerve and subsequent paralysis and loco-regional lymphoganglionization metastases, osteosarcoma of the maxillae, usually the osteolytic form, with the invasion of the periosteum, osteomyelitis (Gougerot) and metastasizing on its blood (lung, etc.) or osteofibrosarcoma with periosteal starting point and association with the cartilage-like ccondrosarcoma, under its different forms hyaline or fibrous and with a vascularization that represents besides the cellular atypia, a characteristic of the malignancy of the respective tumor process. Ewing tumor, which rarely occurs in the jaws, especially in young people, with predominant localization at the level of the mandible. Microscopically, the tumor consists of small, round, uniform, small cytoplasmic tumor cells containing PAS-positive glycogen.

The cells present a round nucleus, with obvious nucleolus, and cell boundaries are indistinct. The tumor has areas of necrosis and hemorrhage. Rare, muscular (tongue, masseter) tumors: myoblastoma (Abrikosov) with possibility of malignancy.

In addition to lymphatic and hematogenous metastasis, we must also mention the direct (mechanical) suction pathway in the gastrointestinal and respiratory tract; from the gastrointestinal tract to reach the liver through the portal vein.

In connection with the rich network of lymphatic pathways and lymph nodes, the lymphoanglionic tumors, such as the Hodgkin's and Neo-Hogkin's malignant lymphomas, there are frequent locations; and they include the adenopathies of leukemias, when even early gingival localization of leukotic infiltrates is noticed, sometimes in the masseter muscle, a clinical aspect being realized in the respective region.

Let's remind, the malignant hemangiopericitoma with local invasion and metastasis itself on the line of the respective vessels; the same for hemangiosarcoma, with extremely severe evolution, affecting the soft or hard parts.

The complications that appear for some of these tumors are very numerous, depending on their shape and locations, with feeding, phonation difficulties.

The metastases that may occur in the oral region, starting with primary neoplasms (in the mandible, or the soft parts) will also be noted. At the level of the mandible, these metastatic nodules may necrosis, producing cysts with spontaneous fractures.

\section{Experimental part}

Material and method

Our study includes 112 patients, who were sent by dentists, family doctors, dermatologists, ENT specialists, BMF surgeons. All cases were from Galați County.

\section{Results and discussions}

In incidence, algal syndromes were found 25 cases (22.32\%) viral, bacterial and fungal infections 32 cases $(28.56 \%)$, oral lichen 18 cases $(16.68 \%)$ out of 14 cases $(12.50 \%)$, benign and malignant tumors 8 cases $(7.12 \%)$ lesions with malignant potential 6 cases $(5.35 \%)$, salivary gland disease 2 cases $(1.78 \%)$ dermatoses, 2 cases $(1.78 \%)$, gingival hyperplasia 3 cases $(2.67 \%)$ allergic reactions 2 cases $(1.78 \%)$. There have also been oral manifestations of rare conditions, such as oro - facial granulomatosis ,

Extremely varied tumors in form and evolution come from multiple undifferentiated or differentiated tissues, often in a tangle of different structures, which results in a histological polymorphism.

They can be detected and tracked in their evolution more easily than those that develop at the level of the organs, at the dental examination. 
An early detection of the precancerous conditions and of the neoplasms themselves can be made, requiring a corroboration of the clinical and paraclinical data, in the dynamics of the evolutionary process, with the mention that in the presence of benign histological aspects, the evolution can be serious through the functional disturbances ( lymphangioma of the tongue with atrophy of the muscle fibers, ulceration of the epithelium and inflammation, with the phlegmon of the buccal floor), hemorrhages (hemangioma of the oral mucosa).

\section{Conclusions}

To maintain oral health dentist needs to know and treat these diseases of the oral mucosa.

In order to develop effective prevention and treatment measures when needed, mucosal disorders should be understood in terms of their distribution, etiology and epidemiological characteristics.

\section{References}

1.IORDAN-DUMITRU AD, DAVID E, DARAGIU DE, Antipa C- Morphology and semiology of the decisional tooth, Printech Publishing House, Bucharest, 2011.

2.BOBOC G- The dentomaxillary apparatus - training and development. Edition of II. Medical Publishing House, Bucharest, 2016:

3.REGEZI JA, SCIUBBA JJ, JORDAN RCK- Oral pathology-clinical pathologic correlations, 5th, Saunders Elsevier 2008.

4.DIRTU, D., LUNGU, N.C., CHIRITA, P., et al.,Synthesis of Novel 4-(3,5-Dibromo-2-hydroxyphenyl)-5-Methyl-1,3-Dithiol-2-ylidene Derivatives, Rev.Chim.(Bucharest) ,67, no. 3 , 2016, p. 534-537

5.CHECHERITA, L. E., FORNA, N.C., MACOVEI, SURDU, A. et al.Influence of Chemical Therapeutical Methods on ManducatoryMuscles, Rev.Chim. (Bucharest), 64, no.11, p. 1312-1316,2013

6.SANDEEPA, NC, et al. "Prevalence of oral mucosal lesions among Pre-University students of Kodava population in Coorg District." Journal of international oral health: JIOH 5.3 (2013): 35.

7.DOS SANTOS, PAULO JOSÉ BENEVIDES, et al. "Cross - sectional study of oral mucosal conditions among a central Amazonian Indian community, Brazil." Journal of oral pathology \& medicine 33.1 (2004): 7-12.

8.DASCALU, C.G., ANTOHE, M.E., GOLOVCENCU L., et al.,Interaction Schemes for the Analysis of Combined Action of Risk Factors, 2017 IEEE International Conference on e-health and Bioengineering Conference (EHB) Book Series: E-Health and Bioengineering Conference, 2017, Pages: 462-465

9.EARAR, K., GRADINARU,S., PARIZA,G., et al., Effect of the Sterilization Procedures of Different Surgical Meshes for Abdominal Surgery, Rev.Chim.(Bucharest), 68, no. 8, 2017, p. 1868-1873

10.CIOCAN-PENDEFUNDA,A.A.,MARTU,M.A.,ANTOHE,M.E., et. al.,Indirect composite veneers as a social therapeutic solution.A case report, Romanian Journal Of Oral Rehabilitation, 10 (4), 2018 Pages: 91-96

11.GRADINARU, I., ANTOHE M.E., HURJUI, L.L, Biomaterials used in oral rehabilitation of the edentulous allergic patients, Romanian Journal of Oral Rehabilitation,10(1),2018, Pages: 114-119

12.SCUTARIU, MIHAELA MONICA; CIURCANU, OANA ELENA; FORNA, DORIANAAGOP; et al.,Importance of dental maxillofacial aesthetics in dental therapy, MedicaL-SurgicalJournal Volume: 120 Issue: 4 Pages: 926-931 Published: 2016

13.STOIAN, A.; EARAR, K.; BUDACU, C., et al.,No Association Between Antioxidant Enzyme Gene Polymorphism and Albuminuria in Type 2 Diabetes Mellitus Cases, Rev.Chim.(Bucharest), 67 , no. 11 , 2016, p. 2355-2359

14.Țovaru Ș, Țovaru M, Costache M, Demarosi F, Mihai L- Medicine and oral pathology. Qmed Publishing SRL, Bucharest, 2012.

Manuscript received: 26.11 .2019 